\title{
Individual differences and interrelationships among a select set of cognitive skills
}

\author{
ALICE CHIANG and RICHARD C. ATKINSON \\ Stanford University, Stanford, California 94305
}

\begin{abstract}
An experiment was conducted to investigate individual differences and interrelationships in performance on three short-term memory processing and visual processing tasks. Parameters of models for these tasks were correlated. High correlations (.97 and .83) were obtained for some intertask parameters, indicating that elemental component processes for different tasks can be identified that are similar or highly related. Psychometric measures (SATM and SATV) were also correlated with the information processing model parameters. High multiple correlations of SATV and SATM were obtained using model parameters as predictors, when the data were analyzed separately for female and male subjects. The results are suggestive of sex differences in the interrelationships of the cognitive processes under investigation.
\end{abstract}

Developments in cognitive psychology over the last 10 years have defined a general theoretical framework within which it is possible to identify cognitive structures and processes which underlie cognitive behavior. These theories are formulated in the language of information processing models. The human mind is viewed as an information processor that accepts environmental input and transforms these informational units by such processes as coding, storage, elaboration, retrieval, and concatenation to produce cognitive behavior (Neisser, 1967).

Within this framework, data from laboratory studies of specific cognitive tasks have been applied by experimental psychologists to the formulation of models of behavior, from models of simple perceptual processes to those of more complex problem-solving tasks such as chess playing (Chase \& Simon, 1973). Although the various models differ in detail, there is considerable agreement among them. For example, most models distinguish between storage and retrieval processes, between memory structures and control processes, between short- and long-term memory, between semantic and episodic representations in long-term memory (See Anderson \& Bower, 1973; Atkinson \& Juola, 1974; Atkinson \& Shiffrin, 1968; Norman \& Rumelhart, 1970; Wescourt \& Atkinson, 1976).

How are the component processes of models for different tasks related to one another? For example, can the rate of visual encoding be predicted from the rate of auditory encoding? One approach to this investigation is the comparison of individual's performances on tasks which seem to have similar or related component processes. Individual differences in cognitive behavior have not traditionally been of primary concern

Dr. Chiang is currently with RMC Research Corporation, Mountain View, California. to experimental psychologists interested in building general models of behavior (Cronbach, 1957). However, as questions concerning the interrelationships among components of the cognitive system emerge, attention is now being directed toward experimentally investigating individual differences in cognition (Hunt, Frost, \& Lunneborg, 1973; Hunt, Lunneborg, \& Lewis, 1975; Underwood, 1975).

In addition to theory building, two practical reasons exist for educators and psychologists for the understanding of relationships among cognitive structures and processes. First, the specification of interrelationships among tasks that span the range of human intellectual skills can provide a basis for the development of a test battery to measure cognitive and perceptual performance. Such a measuring instrument can be used for assessing specific effects of various environmental conditions on human cognitive functions; for instance, the effects of drugs, noise, motion, isolation, aging, sleep deprivation, and so forth, on specific cognitive functions such as short-term memory capacity or speed of perceptual encoding. Work on developing a test battery of information processing tasks is just beginning (Rose, Note 1). However, further understanding of the relationships of the parameters of information processing models is needed before a valid and sensitive instrument can be developed.

A second major value of examining individual differences in the parameters of cognitive models is that it can provide an alternative and theoretically sound approach to the measurement of different human cognitive abilities. Since Binet, psychometricians have been constructing empirical measurements of individual differences in human cognitive ability. However, these aptitude tests have been developed without the support of a theory of cognition. The atheoretical nature of intelligence tests has posed questions of construct validity of these tests. With the emergence of an infor- 
mation processing theory of cognition, cognitive measures of psychometric tests can be reexamined in terms of the structures of memory and processes of cognition identified by a cognitive theory.

Recently, several efforts have been directed toward establishing a link between psychometric tests and cognitive information processing theory. Studies by Hunt et al. $(1973,1975)$ and Lunneborg (Note 2) have correlated and factor analyzed a large array of information processing task measures and psychometric test measures. As a result, they have begun to describe a profile of a high verbal ability person in terms of parameters of a set of cognitive processes (Hunt et al., 1975). Another rapproachment between psychometricians and cognitive psychologists has been offered by Carroll (1976), who has characterized factors of intelligence tests in terms of their cognitive information processing requirements. A third approach to this research is offered by R. Sternberg, who has synthesized psychometric and information processing views into a "componential analysis" approach toward the assessment of cognitive ability (Stemberg, R., 1976). By this approach, psychometric abilities are internally validated by component information processing parameters, and component parameters are in turn externally validated by global cognitive abilities. Although each of the studies cited takes a different approach in its investigation, all are directed toward a common research goal of specifying the relationships among measures that have been developed for describing performance in cognitive tasks. However, findings from this research have not yet demonstrated unequivocal patterns of relationships among psychometric intelligence measures and parameters of information processing models.

We propose here another method for approaching this research goal. We begin by limiting our selection of cognitive tasks to a few well investigated, replicable ones, involving processes that are theoretically related, although experimentally undemonstrated. Large amounts of data for each task measure are collected from the same subjects over several sessions of carefully controlled experimentation. In this way, we hope to obtain highly reliable data for individual subjects. The task measures are then examined in terms of models that fit the group data as well as differences that exist in individual data. The parameters from these models are then correlated with one another as well as with a psychometric measure to determine their relationships. Through this carefully controlled laboratory examination of a few task measures for individual subjects, we hope to establish the interrelationships among the selected set of cognitive skills.

To begin, we have chosen to examine three laboratory tasks which involve short-term memory (STM) processing and visual processing. These three tasks are (1) a short-term memory search task, (2) a visual search task, and (3) a digit span task. In the memory search task, a set of one to five letters is presented visually and sequentially to the subject, followed by a probe letter. The subject's task is to search the memory list and report whether the probe is contained in the memory set. The dependent measure is the subject's reaction time (RT) from the onset of the probe item to his response. The visual search task is similar to the memory search task paradigm. In this task, a target letter is first displayed, followed by a visual display set of one to five letters which appear simultaneously. The subject searches the display list and reports whether the target letter appears in the visual display set. Again the subject's latency is recorded. In the digit span task, a memory set of 4-12 digits is presented sequentially to the subject. The subject's task is to recall the items in the order of their presentation.

Three major considerations were given to the task selection. First, these tasks are well investigated ones, for which models of performance exist, and the model parameters theoretically correspond to specific cognitive processes. The STM search task was developed by S. Sternberg $(1966,1969)$ and has become a paradigm for RT recognition memory studies (Wescourt \& Atkinson, 1976). From such memory experiments, when learning and retention are perfect, it is possible to estimate memory search and retrieval time by varying the number of elements in the memory list. Numerous studies employing this paradigm have shown that RT increases linearly with memory set size. Sternberg has interpreted this function in terms of four separate stages of processing. The slope of the RT vs. set-size function is the time of a single comparison between the probe and an item in the memory set. The intercept is the time required for all processes other than memory search, that is, (1) encoding the probe item, (2) deciding whether or not a match has been found, and (3) producing a response. Several studies of the visual search task by Atkinson, Holmgren, and Juola (1969), Estes and Taylor (1964, 1966), and Estes and Wessel (1966) have also shown that response latency in this task is an increasing linear function of the number of display elements. A similar but slightly different interpretation is given to the slope and intercept parameters of the visual search task because in this task an encoding process is required for each additional item in the display set. Thus, the intercept represents the duration of the two processes, (1) binary decision and (2) response production, whereas the slope represents the time for the encoding process, as well as the comparison process, for each item in the display set. Figure 1 presents a hypothetical graphic interpretation of the processes represented by the slope and intercept parameters of the visual search task and the memory search task. The intercept corresponds to the total time of all processes that occur just once, regardless of set size; the slope represents the duration of processes that occur once for each member of the memory or visual set. The third task, the digit span task, is similar to that used in a standard IQ test. The maximum number of items that a person can recall 


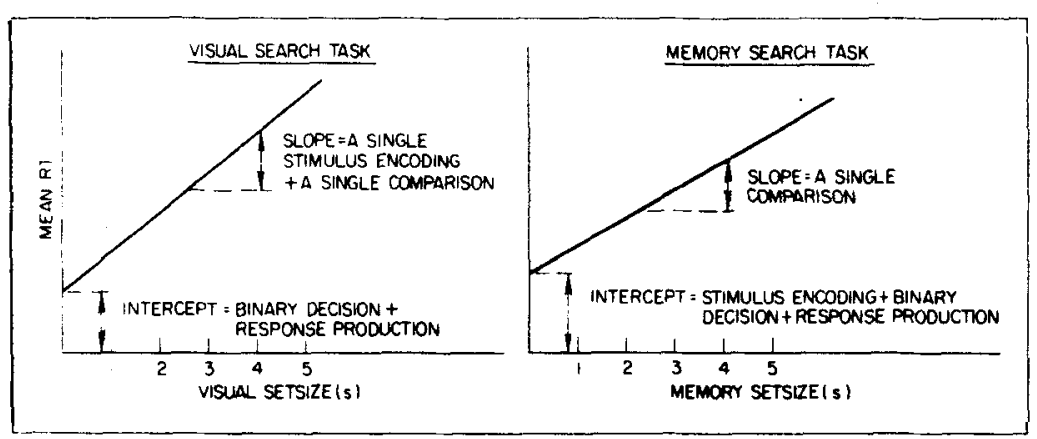

Figure 1. Interpretation of processes represented by the slope and intercept estimates in the memory search and visual search tasks. correctly is theoretically a measure of his STM capacity.

A second reason for selecting these tasks is that they are simple tasks for which prior learning is not necessary, nor is performance dependent on prior experience. Studies with all three tasks have shown that, after initial practice effects, performance becomes stable; thus, reliable estimates can be obtained.

The third reason that these three tasks were selected is that we believe they entail similar or related memory structures and cognitive processes. For example, the slope of the memory search task represents the rate of comparison between a probe item and an item in shortterm memory, whereas the slope of the visual search task represents predominantly the rate of comparison between a target item and an item in a visual display. We want to determine whether visual search rates are related to memory search rates. Similarly, can the intercept from one task be predicted from the intercept of the other? Gilford and Juola (1976) have found no significant differences for the RT and error data between the memory search and visual search tasks. They imply that identical processes are involved in the two comparisons. Ward (Note 3) has shown high correlation between the stimulus coding processes of the S. Sternberg (1966, 1969) task and Posner's (1967) perceptual matching task, thus giving further support to the hypothesis that there is considerable overlap between visual search and memory search processes. Cavanagh (1972) has argued that recall in a memory span task and recognition in a memory search task both access a common memory system; therefore, span and processing rate measures should both reflect parameters of this system store.

The present experiment was conducted to compare performance on these three tasks: memory search, visual search, and digit span. Each subject participated in four sessions, of which the first was a practice session. Subsequently, each session contained balanced blocks of trials for all three tasks. Accuracy and response time measures were collected for each trial. From these data, parameters of models were estimated. In addition to comparing the results of these tasks, Scholastic Aptitude Test (SAT) verbal and quantitative scores were obtained and correlated with the parameters.

\section{METHOD}

Subjects

Thirty-four Stanford University undergraduates $(17$ males and 17 females) served as subjects. All subjects completed the experiment and were paid for their participation.

\section{Apparatus}

The experiment was conducted on a programmable display system (Imlac Corporation PDS-1) interfaced with a PDP-10 timesharing computer system. All stimuli presentation, trial generation and sequencing, response recording and feedback were controlled by the computer. The PDS- 1 was located in a laboratory room in the Department of Psychology at Stanford University. Subjects viewed stimulus materials on the cathoderay tube (CRT) screen (approximately $24 \times 29 \mathrm{~cm}$ ) from a distance of approximately $50 \mathrm{~cm}$. Subjects responded through a typewriter keyboard with microswitch keys that was located in front of the CRT screen.

\section{Procedure}

Each subject participated in four approximately 1-h sessions. The sessions were scheduled on 4 separate days, spaced over 1 week. During the first session, subjects received instructions and then practiced on one block of trials. One block of trials consisted of 50 memory search trials, 50 visual search trials, and 25 digit span trials. Subjects were asked to respond as quickly as possible without making errors. On each of the three remaining sessions, subjects began by warming up on a set of practice trials. Subsequently, they were given two complete blocks of trials. From these three sessions, data for a total of 300 memory search, 300 visual search, and 150 digit span trials were recorded for each subject.

\section{Task Description}

Memory search task. In this task, memory set items of one to five consonant letters were presented sequentially, followed by a probe letter. The subject's task was to decide whether the probe was contained in the memory set. Performance on this task is essentially error free; the dependent variable is the subject's RT, measured in milliseconds from the onset of the probe to the time when the subject makes a response. The probe letter may be positive (contained in the memory set) or negative (not contained in the memory set). The memory set size varied from one to five items. Thus, there were a total of 10 trial types, characterized by five memory set sizes and either a positive or negative probe. Within a block of 50 memory search trials, each trial type occurred equally often (i.e., five times). Serial positions of correct responses were also balanced for each trial type. At the onset of every trial, the computer program randomly generated, from the stimuli ensemble of 20 consonants, the probe item and the items in the memory set, with no two memory set items being the same. The order of trial presentation was randomized within a set-order constraint (see below). Thus, for each subject and each block, there was a different sequence of trials.

The following sequence of events occurred on each memory search trial: (1) At the beginning of each trial, a ready signal identifying the task ("Memory Search") was displayed. Each subject initiated the trial by pressing the space bar, indicating that he was ready. (2) Subsequently, a warning character ("\#") appeared at the center of the CRT screen, followed by a sequential presentation of the memory set letters, terminated by a 
memory set delimiter character ("*"). Each character appeared in the same centered position for $800 \mathrm{msec}$, with a $200-\mathrm{msec}$ wait between characters. (3) Two seconds later, the probe letter was displayed four lines directly below the "*”. (4) Subjects responded by pressing either the "." key with the right index finger for a positive response or the " $x$ " key with the left index finger for a negative response. Subjects were instructed to place their index fingers on these keys throughout the trials, so as to eliminate additional response time for key search. (5) The Imlac PDS-1 computer recorded the response and its latency to the nearest millisecond. A time-out or error message was displayed for $2 \mathrm{sec}$ if subjects failed to answer within $5 \mathrm{sec}$ or made a wrong response. (6) The experiment continued to the next trial. Each trial lasted approximately $5-10 \mathrm{sec}$.

Visual search task. In this task, a target letter was first presented, followed by a linear display of one to five consonant letters. The subject's task was to decide whether the target letter was in the display set. Since the specification for this task is completely analogous to the memory search task, the reader is asked to refer to the above description of the memory search task for information on the dependent measures, the trial types, the assignment of trials to blocks, the order of trial presentation, the selection of display and target items, and so forth.

The sequence of events for a visual search trial also corresponded to that of the memory search trial. First, a ready signal identifying the task ("Visual Search") was displayed,and the subject initiated the trial by pressing the space bar. Then a warning signal ("\#"), followed by the target letter, was presented for $800 \mathrm{msec}$ with a $200-\mathrm{msec}$ wait between them. Subsequently, a display set warning character ("*") appeared, followed $2 \mathrm{sec}$ later by the display set of one to five letters, which were centered on the screen and four lines below the "**" The display items were separated by a space of about $5 \mathrm{~mm}$; the largest display was approximately $5.5 \mathrm{~cm}$. Subjects responded by pressing the yes and no keys. Their responses were processed in the same manner as in the memory search task.

Digit span task. In this task, memory set items of 4-12 digits were presented sequentially. The subject's task was to recall the digits in the order of their presentation. Digit span trials were grouped in a series of five trials, where the set size of each series progressed from 4 to 12 , with increments of 2 . At the onset of each trial, the computer program randomly generated digits from 1 to 9 for the memory set items, with the constraint that no two adjacent digits were the same. The dependent variable for this task was the number of correct responses for each trial. A response was counted as being correct if it was in the same serial position in the response set as it was in the memory set.

The sequence of events for each digit span trial was as follows: (1) At the beginning of each trial, a ready signal identifying the task ("Digit Span") was displayed on the screen. The subject initiated the trial by pressing the space bar, causing the ready signal to disappear. (2) Subsequently, a warning signal ("\#") appeared at the center of the screen, followed by a sequential presentation of the memory set digits, and terminated by a delimiter character ("*"). Each of these characters appeared in the center position of the CRT screen for $800 \mathrm{msec}$, with a 200-msec delay between them. (3) Next, a line containing the same number of blanks as the number of the memory set items appeared on the screen. As the subject typed back his response, his response was echoed in the appropriate blank space. The subject typed a carriage return to terminate his response. The computer program recorded and evaluated the response. (4) A feedback of the number of correct digits and the number of digits in the memory set was displayed for $2 \sec$ before the program proceeded to the next trial.

Distribution of tasks into sets of trials within a block. During each experimental session, a subject was given two blocks of trials. Each block consisted of 50 memory search trials, 50 visual search trials (balanced for trial types), and five series of digit span trials. These 125 trials were grouped into five sets, where each set contained one series of digit span trials, followed by either 10 memory search trials or 10 visual search trials, followed by 10 of whichever search task had not yet been presented in the set. The tasks were presented in this set-order sequence until all 125 trials in the block had been presented. At the end of each set of memory search and visual search trials, a feedback containing (1) the current number of errors for the task in the current block, (2) the average RT per item for the current block, and (3) the average RT per item for all previous blocks was displayed. These cumulative result feedbacks are not standard procedures for such experiments. They were included here so that subjects would be aware of their own performance. In this way, we believed subjects would pay closer attention to the task, and thus minimize their error rate and maintain a consistently fast response time.

In studies such as the present one, it is important to arrange the experimental conditions so as to maximize the probability of obtaining reliable performance data. To this end the experiment was designed (1) to reduce the practice effects on performance by giving subjects ample practice trials, (2) to equalize conditions for all trials, tasks, sessions, and so forth, by utilizing balance assignments and using randomization wherever possible, and (3) to insure that subjects are attending the tasks by allowing subjects to control the intertrial interval, and by giving subjects cumulative feedback of their performance.

\section{RESULTS}

\section{Group Performance on Tasks}

The data were first analyzed for group results. For the two search tasks, mean RTs for correct responses as a function of memory or visual set size are presented in Table 1, along with error rates and SDs for the 34 subjects. The group mean RTs were computed as an average of individual mean RTs. Outliers were first discarded from each subject's data. For any subject $i$ and and any trial type $\mathrm{j}, \mathrm{RT}_{\mathrm{ij}} \geqslant \overline{\mathrm{X}}_{\mathrm{ij}}+3 \mathrm{SD}_{\mathrm{ij}}$ were counted as outliers. The mean percent of outliers per subject was $1.7 \%$ and the mean error rate was $3.4 \%$, totaling about $5 \%$ of an individual's data that were discarded. Thus, the group mean RT is based on approximately $95 \%$ of the total data or approximately 970 observations per data point. Figure 2 contains plots of (1) mean latencies of correct positive and negative responses as a function of set size, and (2) mean latencies for correct positive responses as a function of serial position of the target item.

The results in Table 1 and Figure 2 are similar to S. Sternberg's (1969) results for the memory search task and the findings of Atkinson et al. (1969) for the visual search task. Namely, (1) RT increases linearly with set size, with the exception of $s=1$; (2) negative RT is greater than positive RT, but the slopes of the negative and positive responses appear to be about the same; (3) serial position curves are relatively flat. These results seem to be explained well by a serial-exhaustive scanning model (Atkinson et al., 1969; S. Sternberg, 1969; Wescourt \& Atkinson, 1976). Adopting this theoretical model, two parameters, the slope and intercept, can be estimated. Because Set Size 1 did not conform to a linear relationship with the other set sizes, the data for $s=1$ were not included in this estimation. 
Table 1

Mean RT and SD for Correct Responses and Mean Percentage Errors for Positive and Negative Responses as a Function of Memory Set Size for the Memory Search Task and as a Function of Display Set Size for the Visual Task, for 34 Subjects

\begin{tabular}{|c|c|c|c|c|c|c|}
\hline \multirow[b]{2}{*}{$\begin{array}{c}\text { Set } \\
\text { Size }\end{array}$} & \multicolumn{3}{|c|}{ Negative } & \multicolumn{3}{|c|}{ Positive } \\
\hline & $\begin{array}{c}\text { Mean } \\
\text { RT } \\
\end{array}$ & SD & $\begin{array}{c}\% \\
\text { Errors }\end{array}$ & $\begin{array}{c}\text { Mean } \\
\text { RT }\end{array}$ & $\mathrm{SD}$ & $\begin{array}{c}\% \\
\text { Errors }\end{array}$ \\
\hline & \multicolumn{6}{|c|}{ Memory Search Task } \\
\hline $\begin{array}{l}1 \\
2 \\
3 \\
4 \\
5\end{array}$ & $\begin{array}{l}496 \\
529 \\
578 \\
615 \\
649\end{array}$ & $\begin{array}{l}113 \\
110 \\
108 \\
106 \\
120\end{array}$ & $\begin{array}{l}1.9 \\
1.2 \\
1.1 \\
3.1 \\
4.2\end{array}$ & $\begin{array}{l}444 \\
502 \\
556 \\
590 \\
640\end{array}$ & $\begin{array}{l}112 \\
100 \\
119 \\
140 \\
154\end{array}$ & $\begin{array}{l}1.2 \\
3.4 \\
3.9 \\
3.9 \\
7.0\end{array}$ \\
\hline 5 & \multicolumn{6}{|c|}{ Visual Search Task } \\
\hline $\begin{array}{l}1 \\
2 \\
3 \\
4 \\
5\end{array}$ & $\begin{array}{l}545 \\
543 \\
580 \\
628 \\
719\end{array}$ & $\begin{array}{l}128 \\
105 \\
124 \\
116 \\
145\end{array}$ & $\begin{array}{l}4.2 \\
2.3 \\
2.1 \\
3.0 \\
3.0\end{array}$ & $\begin{array}{l}458 \\
493 \\
524 \\
563 \\
610\end{array}$ & $\begin{array}{l}106 \\
103 \\
106 \\
113 \\
133\end{array}$ & $\begin{array}{l}1.3 \\
2.2 \\
4.3 \\
5.7 \\
5.6\end{array}$ \\
\hline
\end{tabular}

To estimate the slope and the intercept for both positive and negative responses, (1) the average difference $d$ between positive and negative responses as a function of set size was computed and added to the positive means; (2) the slope was estimated by fitting the line that minimized the squared deviation for the negative and adjusted positive means; (3) the intercept from this estimation was decreased by $d / 2$ to obtain the average intercept for positive and negative responses. The obtained slope and intercept for the memory

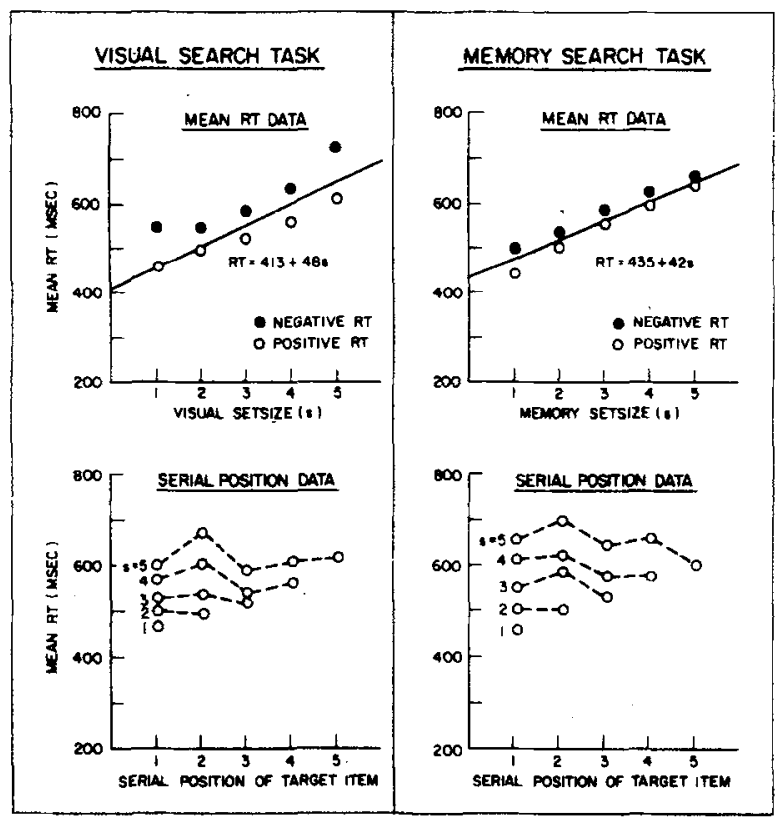

Figure 2. Mean RT for correct positive and negative responses as a function of set size. Line fitted by least squares to the average adjusted negative and positive means for Set Sizes 2-5 (top panels). Mean RT for positive responses as a function of serial position of the target item in the memory or visual set (bottom panels).

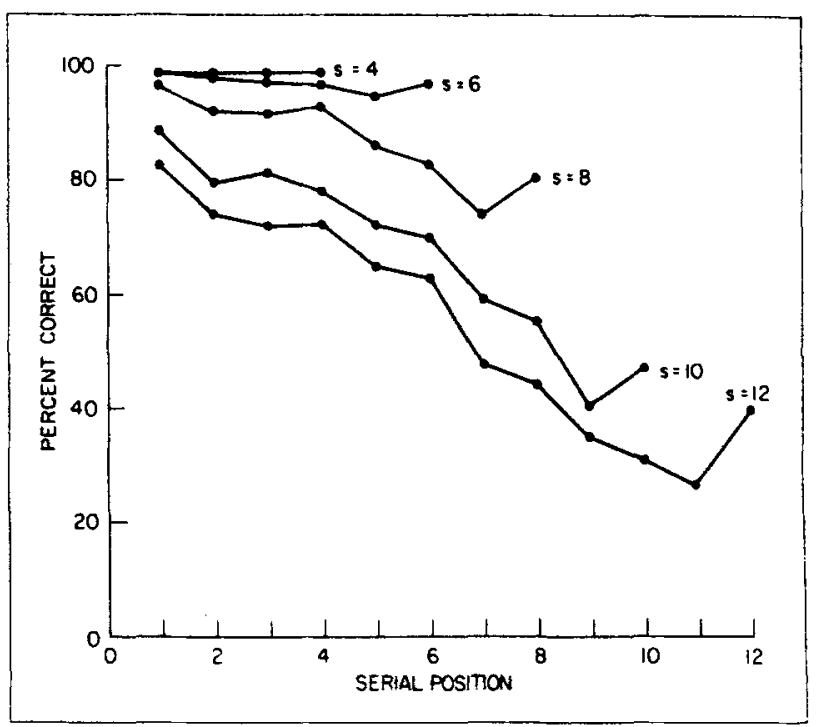

Figure 3. Mean percent correct in the digit span task as a function of serial position, for Set Sizes 4, 6, 8, 10, 12 .

search task were 42 and 435 , respectively; the slope and intercept for the visual search task were 48 and 413. Confidence bands were obtained for the slopes. For the memory search slope, the confidence interval was $42 \pm 6.6$; for the visual search slope, it was $48 \pm 8.8$, for $\alpha=.05$. Thus, no significant differences exist between the slopes of the two tasks. This finding concurs with that of Gilford and Juola (1976). However, the slope obtained for the visual search task is larger than that obtained by Gilford and Juola. For both tasks, the group mean RT data and serial position data conform to the hypothesized models of group performance from other studies of these tasks.

An examination of the group data for the digit span task also indicates that these results are in agreement with the findings of other memory span studies. Figure 3 is a plot of the mean percent correct as a function of serial position correct for the five set sizes. Each data point is based on 30 observations for each of the 34 subjects, totaling 1,020 observations. As can be observed from Figure 3, the total percent correct decreases as the set size increases, and for all set sizes, there is a primacy and slight recency effect.

\section{Task Parameters for Individuals}

To examine individual differences in performance, two parameters were estimated for each task, for each subject. For the memory search task, a slope (MSLOPE) and an intercept (MINT) were estimated, and for the visual search task, a slope (VSLOPE) and an intercept (VINT) were estimated, for each individual subject's data, in the same way that the slope and intercept were estimated for the group data. One dependent measure for memory span was the average largest set size of complete/correct (MSPAN), computed in the following way: (1) From each series of five digit span trials, the largest set size in which the subject recalled the 
Table 2

Descriptive Measures for Task Parameters and SAT Scores

\begin{tabular}{|c|c|c|c|c|c|c|c|c|c|}
\hline \multirow[b]{2}{*}{ Parameters } & \multicolumn{4}{|c|}{ All Subjects $\dagger$} & \multicolumn{2}{|c|}{ Female Subjects $\dagger \dagger$} & \multicolumn{2}{|c|}{ Male Subjects $\dagger \dagger$} & \multirow{2}{*}{$\begin{array}{c}\mathbf{t} \text { Test of } \\
\text { Differences } \\
\text { in Means }\end{array}$} \\
\hline & Mean & SD & Min & $\operatorname{Max}$ & Mean & SD & Mean & SD & \\
\hline MINT & 484 & 102 & 301 & 774 & 523 & 114 & 445 & 72 & 2.0 \\
\hline MSLOPE & 43 & 20 & 12 & 117 & 49 & 25 & 37 & 12 & 1.62 \\
\hline VINT & 472 & 83 & 347 & 696 & 504 & 90 & 440 & 62 & $2.19 *$ \\
\hline VSLOPE & 42 & 21 & 12 & 103 & 49 & 26 & 36 & 12 & 1.70 \\
\hline MTOT & 28.6 & 6.3 & 22.9 & 35.7 & 28.5 & 2.7 & 28.6 & 8.6 & .17 \\
\hline MSPAN & 7.3 & .9 & 5.7 & 9.3 & 7.0 & .8 & 7.6 & 1.0 & 1.50 \\
\hline SATV & 613 & 79 & 430 & 760 & 608 & 74 & 618 & 85 & .33 \\
\hline SATM & 660 & 77 & 480 & 790 & 624 & 78 & 697 & 62 & $2.72 *$ \\
\hline
\end{tabular}

entire stimulus set correctly (LSSC) was recorded; (2) MSPAN is the average LSSC over 30 digit span series for each subject. The other digit span variable was the average total number of correct responses (MTOT) for a series of five digit span trials. The maximum MTOT would be 30. SAT verbal (SATV) and SAT math (SATM) scores were two additional measures obtained for each subject. Because the SATV and SATM scores for four subjects (two males and two females) were unavailable, the data for these four subjects were excluded from further analyses.

Table 2 contains the mean and SD for these eight measures, for all subjects, and separately for male and female subjects. As can be observed from Table 2, there is a large SD and large range for all measures, indicating large individual differences in performance. Although the male subjects performed better than the female subjects on almost all the tasks, a t test of differences in means indicates only significant differences for VINT and for SATM variables. The higher SATM scores for the males, with no differences in the SATV scores for males and females, concur with the findings of Bieri, Bradburn, and Galinsky (1958) for a similar college population.

The values of the slopes and intercepts for the individual data are different from values of the slopes and intercepts for the group data (compare Figure 2 with Table 2). In other words, the slopes of the group mean RTs are different from the means of the individual slopes. However, the latter fall within the confidence band of the group slopes. The group parameters were computed so that they could be compared with group results of other studies. However, since this study is

Table 3

Reliability Coefficients for Task Parameters $(\mathrm{N}=30$ )

\begin{tabular}{|c|c|c|c|}
\hline \multirow[b]{2}{*}{ Parameter } & \multirow{2}{*}{$\begin{array}{l}\text { Split-Half } \\
\text { Blocks } 1,3,5 / \\
\text { Blocks } 2,4,6\end{array}$} & \multicolumn{2}{|c|}{ Test-Retest } \\
\hline & & $\begin{array}{c}\text { Day 1/ } \\
\text { Day } 2\end{array}$ & $\begin{array}{l}\text { Day } 2 / \\
\text { Day } 3\end{array}$ \\
\hline MINT & .96 & .73 & .81 \\
\hline MSLOPE & .89 & .28 & .78 \\
\hline VINT & .95 & .70 & .80 \\
\hline VSLOPE & .91 & .29 & .70 \\
\hline MTOT & .95 & .85 & .79 \\
\hline MSPAN & .95 & .87 & .85 \\
\hline
\end{tabular}

interested in examining individual differences, the individual parameters are used in all subsequent analyses.

\section{Reliability of Task Parameters}

For each subject, six blocks of data were collected over 3 separate days. Table 3 shows the split-half and the test-retest reliabilities for the task parameters. The splithalf reliabilities were calculated by applying the Spearman-Brown formula to the combined data of Blocks 1, 3, 5, and Blocks 2, 4, 6. The split-half reliabilities for all tasks are high, indicating that subjects performed consistently across blocks when the effects of days had been controlled. The test-retest reliabilities were calculated by using the Pearson product-moment correlation formula for data from Day 1 with that from Day 2, and for data from Day 2 with that from Day 3. All the correlations were high, except for the slope parameters for Days 1 and 2. This low correlation for the slopes between Days 1 and 2, but subsequent higher correlation between Days 2 and 3, indicate that there is a large practice effect on the slopes and that these parameters require more practice trials before they become stable. This result is consistent with what Rose (Note 1) found for the test-retest reliability for the slope of a similar task. Otherwise, all task parameters show high reliability.

\section{Correlation Among Parameters}

Table 4 presents the correlation among the six task parameters and the SAT scores. As can be observed from this table, the correlation between the intercepts of the memory search and the visual search tasks is .968; the correlation between the slopes of these two tasks is .832 . However, within the same task, the slopes and intercepts correlated .107 for the memory search task and --.286 for the visual search task.

Figures 4 and 5 are scatter plots of MINT with VINT and MSLOPE with VSLOPE for each of the 30 subjects. Lines are fitted by least squares to the 30 observations, and regression equations are presented. The slope of the visual search task is greater than that of the memory search task (MSLOPE $=7+.83$ VSLOPE), whereas the intercept of the memory search task is greater than that of the visual search task (MINT $=-72+1.18$ VINT).

Because the test-retest reliability for the slope para- 
Table 4

Correlation Between Task Measures and SAT Scores for all Subjects $(\mathrm{N}=30$ )

\begin{tabular}{|c|c|c|c|c|c|c|c|c|}
\hline & MINT & MSLOPE & VINT & VSLOPE & MTOT & MSPAN & SATV & SATM \\
\hline MINT & 1.00 & & & & & & & \\
\hline MSLOPE & .107 & 1.00 & & & & & & \\
\hline VINT & $.968 * *$ & .038 & 1.00 & & & & & \\
\hline VSLOPE & .427 & $.832 * *$ & -.286 & 1.00 & & & & \\
\hline MTOT & -.002 & .132 & -.034 & .151 & 1.00 & & & \\
\hline MSPAN & -.231 & .037 & -.352 & -.078 & $.456 * *$ & 1.00 & & \\
\hline SATV & .202 & .194 & .140 & .345 & -.250 & -.179 & 1.00 & \\
\hline SATM & -.389 & .029 & .287 & -.048 & -.118 & -.088 & $.440^{*}$ & 1.00 \\
\hline \multicolumn{3}{|c|}{$N=30, d f=28$} & & $* p<.05$ & & & ${ }^{*} p<$ & \\
\hline
\end{tabular}

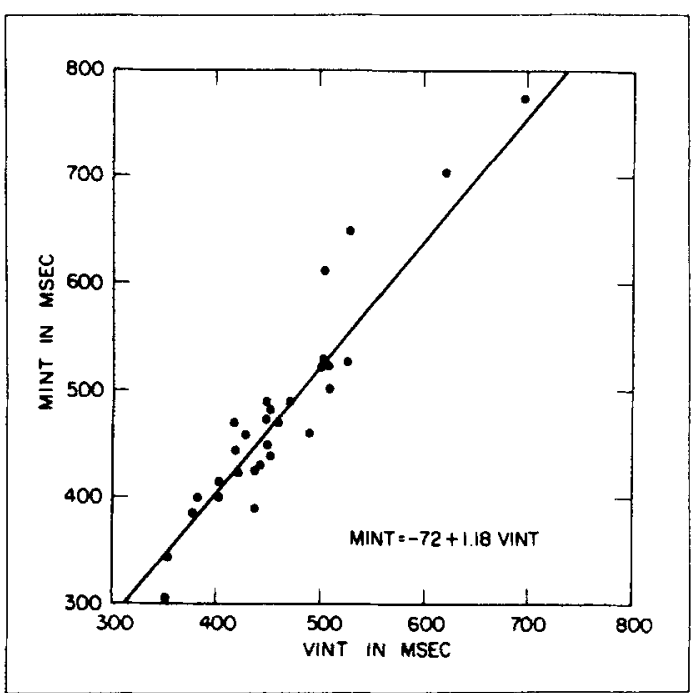

Figure 4. Scatter plot for intercept of memory search task and intercept of visual search task. Every point represents one subject's intercept parameter. $N=30$.

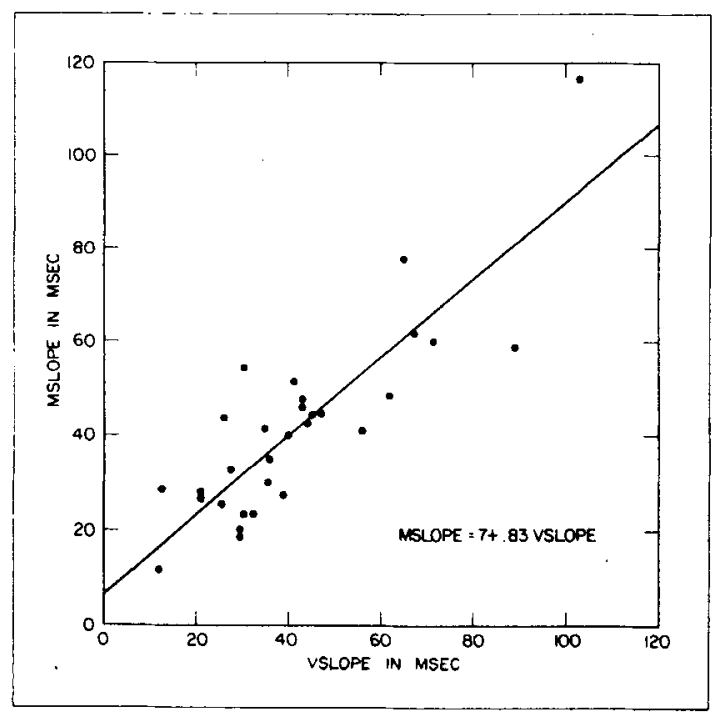

Figure 5. Scatter plot for slope of memory search task and slope of visual search task. Every point represents one individual's slope parameter. $\mathbf{N}=\mathbf{3 0}$. meters was low for Days 1 and 2 (see Table 3), the slopes were also computed using data from Blocks 3-6 (or Days 2 and 3). The MSLOPE and VSLOPE correlation from this data is .892 . The regression equation remained the same (MSLOPE $=7+.83$ VSLOPE). This result is in agreement with the results presented in Table 4 and Figure 5, using the slopes computed from all six blocks of data.

Table 4 also indicates significant correlation between the two digit span parameters, MTOT and MSPAN. This result is not surprising, since MTOT and MSPAN are measuring essentially the same thing (i.e., short-term memory capacity) and the measurements were obtained in a similar way. The other significant correlation is between SATV and SATM. All other correlations are not significant.

\section{DISCUSSION}

The results from the present experiment showed impressively high correlations among some intertask parameters representing the processes under investigation. Specifically, high correlations were obtained between the slopes (MSLOPE with VSLOPE) of the memory search and visual search tasks $(\mathrm{r}=.832)$ and between the intercepts (MINT with VINT) of these two tasks $(r=.968)$. We conclude that the cognitive processes associated with the slopes and the intercepts of the two search tasks are highly related or similar.

Referring to Figure 1, which gives a theoretical interpretation of the processes associated with these parameters, we note that the intercepts of the two search tasks represent two similar processes: binary decision and response production. The high correlation between the intercepts give construct validity to these model parameters. More importantly, it gives empirical support to the general concept that there exist some elemental component processes that are common across tasks. Similarly, the high correlation between the visual search slope and the memory search slope indicates that the visual comparison and memory comparison processes are highly related. Although it is interesting to note that one search rate can be predicted from the other with some accuracy, the more significant implication of 
this finding is that it is possible to experimentally isolate component processes for different tasks and demonstrate high interrelationship between them. None of the studies cited earlier has been able to demonstrate this high correlation between component information processing units from different tasks.

Furthermore, we note from Figure 1 that the intercept of the memory search and the slope of the visual search include the time for a common process, namely, stimulus encoding, whereas the slope of the memory search and the intercept of the visual search do not represent the duration of any common process. The correlational results support this theoretical representation. Table 4 shows an almost significant correlation between VSLOPE and MINT (.427) and a low correlation between MSLOPE and VINT (.038).

If the representation in Figure 1 is accurate, then the intercept of the memory search should be greater than the intercept of the visual search, since the memory intercept includes the time for an additional coding process. Looking at the mean data for individuals in Table 2, we note that MINT is $484 \mathrm{msec}$ and VINT is 472 msec. Although MINT is greater than VINT, a t test of differences in the means shows no significant differences $(t=.524)$. Similarly, no significant differences exist between MSLOPE and VSLOPE $(\mathrm{t}=.20)$. The lack of significant differences between these parameters can mean either that the parameters do not represent the duration of different processes or that the duration of the encoding process is so short that it does not contribute significantly to the differences in the parameters. Additional studies are required to adequately explain this finding.

It might be argued that performance on these search tasks is related to a general factor, speed, and that it is not useful to break down performance into several component processes or to distinguish between parameters of these processes. Two findings from the present experiment refute this argument. First, low correlations were obtained between the slope and intercept of the same search task [r(MINT, MSLOPE) $=.107$ and $\mathrm{r}(\mathrm{VINT}, \mathrm{VSLOPE})=-.286]$. Thus, the speed of searching is not related to the speed of producing a binary response or of making a binary decision. Hunt et al. (1973) also found low correlation between the slope and the intercept $(\mathrm{r}=-.38)$ for the memory search task. The low correlational findings between the parameters of the same search task give additional support to S. Sternberg's (1969) theory of independent stages of processing for the RT recognition task.

Second, analysis of the error data also supports the idea of stages of processing that can be separately characterized by the slope and the intercept. For each subject, the total number of errors (ERROR) and the average time for one response (MEANRT) were obtained from the aggregated visual search and memory search data. The ERROR variable was then correlated with SLOPE, INT, and MEANRT for the 30 subjects. The fol- lowing correlations were obtained: $r(E R R O R$, SLOPE $)=$ $-.531, \mathrm{r}$ (ERROR, INT) $=-.157$, and r(ERROR, MEANRT) $=-.349$. The correlation between ERROR and SLOPE is significant at the .01 level. This result shows that errors are more related to a separate process represented by the slope than to the overall response time, or to the other processes represented by the intercept. This result is intuitively convincing, as it seems reasonable that errors should result from searching too quickly, rather than from encoding the stimuli incorrectly or making an incorrect motor response.

There were no other significant correlations found among the other task parameters, with each other or with the SAT scores (see Table 4). Lunneborg (Note 2), Rose (Note 1), and R. Sternberg (1975) all obtained similar low correlations between the psychometric measures and information processing parameters which they examined. These low correlations discourage making any statements about the relationship between psychometric tests and cognitive information processing theory. As in our results, Hunt et al. (1973) did not find a high correlation between mernory search parameters and memory capacity.

\section{SEX DIFFERENCES IN CORRELATION AMONG TASK PARAMETERS AND PSYCHOMETRIC MEASURES}

\section{Results}

Although the present study was not designed to investigate sex differences in cognitive processes, our sample of half female and half male subjects allowed us to examine this question. Correlations between the slopes and intercepts of the two search tasks were obtained separately for the male and female groups. The results were virtually the same as those obtained for the entire group and showed no sex differences. To simplify the subsequent analyses, the task parameters that were highly correlated were either combined or eliminated. Thus, the average of MINT and VINT were computed to form the intercept parameter INT; the average of MSLOPE and VSLOPE were computed to form the slope parameter SLOPE. MSPAN was selected as a measure of memory span capacity and MTOT was eliminated. Thus, the six task parameters were reduced to three task parameters (INT, SLOPE, MSPAN).

Correlations among the three task parameters and the two SAT scores were computed for all subjects and separately for female subjects and male subjects. Table 5 shows these results. The correlation table for all subjects is not different from the results of Table 4 . That is, there were no significant correlations among the task parameters, with each other or with SAT scores.

However, when the correlations were computed for the male and female subjects separately, a very different pattern of correlations emerged. First, for the female subjects, there is a high significant correlation (.715) between the SATV score and the SLOPE; that is, the 
Table 5

Correlation Between Combined Task Measures and SAT Scores

\begin{tabular}{|c|c|c|c|c|c|}
\hline & MSPAN & INT & SLOPE & SATV & SATM \\
\hline & \multicolumn{5}{|c|}{ All Subjects $(\mathrm{N}=30)$} \\
\hline MSPAN & 1.000 & & & & \\
\hline INT & -.306 & 1.000 & & & \\
\hline SLOPE & -.059 & .241 & 1.000 & & \\
\hline SATV & -.179 & .162 & .281 & 1.000 & \\
\hline \multirow[t]{2}{*}{ SATM } & .088 & -.204 & -.041 & $.440^{*}$ & 1.000 \\
\hline & \multicolumn{5}{|c|}{ Female Subjects $(N=15)$} \\
\hline MSPAN & 1.000 & & & & \\
\hline INT & -.022 & 1.000 & & & \\
\hline SLOPE & -.239 & .252 & 1.000 & & \\
\hline SATV & -.251 & .079 & $.715 * *$ & 1.000 & \\
\hline \multirow[t]{2}{*}{ SATM } & -.075 & -.215 & .331 & .400 & 1.000 \\
\hline & \multicolumn{5}{|c|}{ Male Subjects $(N=15)$} \\
\hline MSPAN & 1.000 & & & & \\
\hline INT & $-.543 *$ & 1.000 & & & \\
\hline SLOPE & $.561 *$ & -.234 & 1.000 & & \\
\hline SATV & -.173 & .386 & --.365 & 1.000 & \\
\hline SATM & -.028 & .300 & -.442 & $.554^{*}$ & 1.000 \\
\hline
\end{tabular}

df for all subjects $=28$, df for female or male subjects $=13$ ${ }^{*} p<.05 \quad p<.01$

higher the SATV score, the higher (slower) the search rate as represented by the SLOPE. However, for the males, these two measures are negatively correlated; that is, the higher the SATV score, the faster the search rate. Similar correlations in opposite directions are found between the SLOPE and the SATM scores for male and female subjects. Second, there is a significant positive correlation between SLOPE and MSPAN for the male subjects and, again, a correlation in the opposite direction for the female group. Third, two other significant correlations exist for the male group (SATV with SATM and INT with MSPAN) but not for the female group. Thus, both the measures that show significant correlation and the directions of their correlation vary for the male and female samples.

Differences in correlations between the male and

Table 6

Significant Differences Between Male and Female Groups for Correlation Between the Slope and Other Variables

\begin{tabular}{lccc}
$\begin{array}{c}\text { Measure } \\
\text { Correlated } \\
\text { with Slope }\end{array}$ & $\begin{array}{c}\text { Correlation } \\
\text { for Female } \\
(\mathrm{N}=15)\end{array}$ & $\begin{array}{c}\text { Correlation } \\
\text { for Male } \\
(\mathrm{N}=15)\end{array}$ & $\begin{array}{c}\text { Z Statistic } \\
\text { of } \\
\text { Differences in } \\
\text { Correlation }\end{array}$ \\
\hline & & Blocks 1-6 & \\
SATV & $.715^{* *}$ & -.365 & $3.13^{* *}$ \\
SATM & .343 & -.442 & $2.00^{*}$ \\
MSPAN & -.239 & $.561^{*}$ & $2.15^{*}$ \\
& & Blocks 3-6 & \\
SATV & $.684^{* *}$ & -.324 & $2.87^{* *}$ \\
SATM & .297 & -.260 & 1.42 \\
MSPAN & -.300 & $.814^{* *}$ & $3.55^{* *}$ \\
\hline
\end{tabular}

Note-Slope computed for Blocks 1.6 and for Blocks $3-6$

$$
{ }^{*} p<.05 \quad{ }^{* *} p<.01
$$

female groups were tested for significance. All the correlations between the SLOPE and the other three measures (SATV, SATM, MSPAN) were significantly different for the male and female groups. The average of SATV and SATM scores (a rough measure of general ability) were also correlated with the SLOPE. For the males, $\mathrm{r}=-.448$ and for females, $\mathrm{r}=.646$. Difference in correlation was significant at the .01 level. These results are presented in Table 6. Because the SLOPE from Blocks 3.6 had higher test-retest reliability (see Table 3), the SLOPE parameter was estimated from Blocks 3.6 as well, and correlated with the other measures. As can be observed from Table 6 , the resulting correlations using the SLOPE estimate from Blocks 3-6 are similar to those using the SLOPE estimate from Blocks 1-6. The correlation between the two SLOPE estimates is 857 .

Figure 6 and 7 are scatter plots of the SLOPE with the three other measures, for the male and female subjects. Lines are fitted to the male and female data separately by the method of least squares, and regression equations are presented. As can be observed from these figures and equations, the slopes of these lines are in opposite directions for the male and female groups.

Finally, to further examine the sex differences in the relationship between task parameters and psychometric measures, stepwise multiple regression equations for predicting SATV and SATM scores from the three task parameters were derived for all subjects, for the male subjects, and for the female subjects. The results are presented in Table 7. Again, different results were

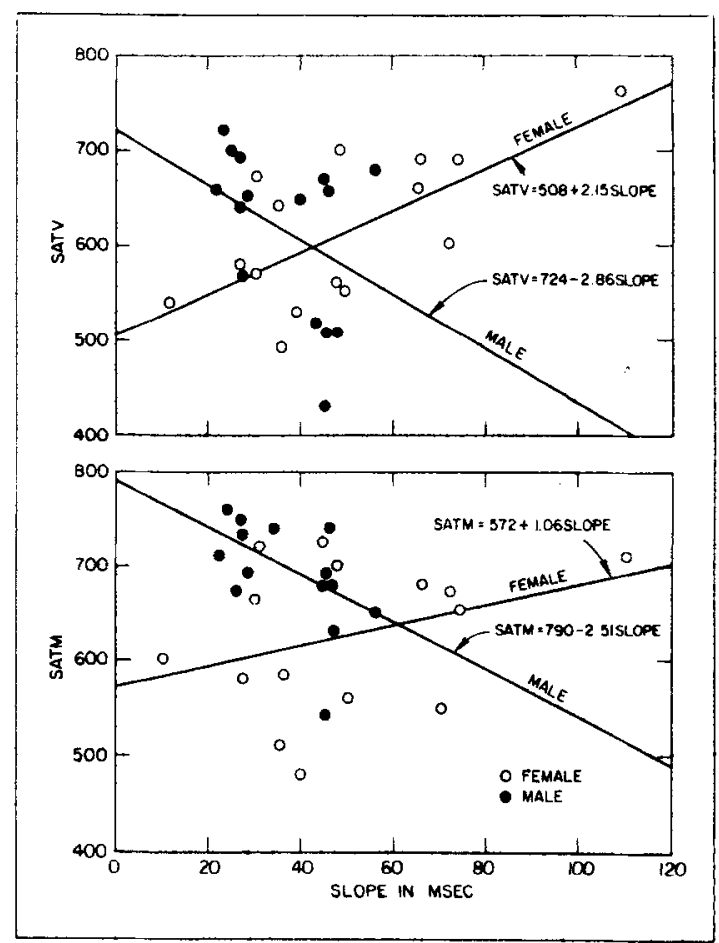

Figure 6. Scatter plot of SATV and SLOPE (upper panei) and SATM and SLOPE (lower panel) for male and female subjects. Lines fitted by least squares to the observations for male subjects and for female subjects. 


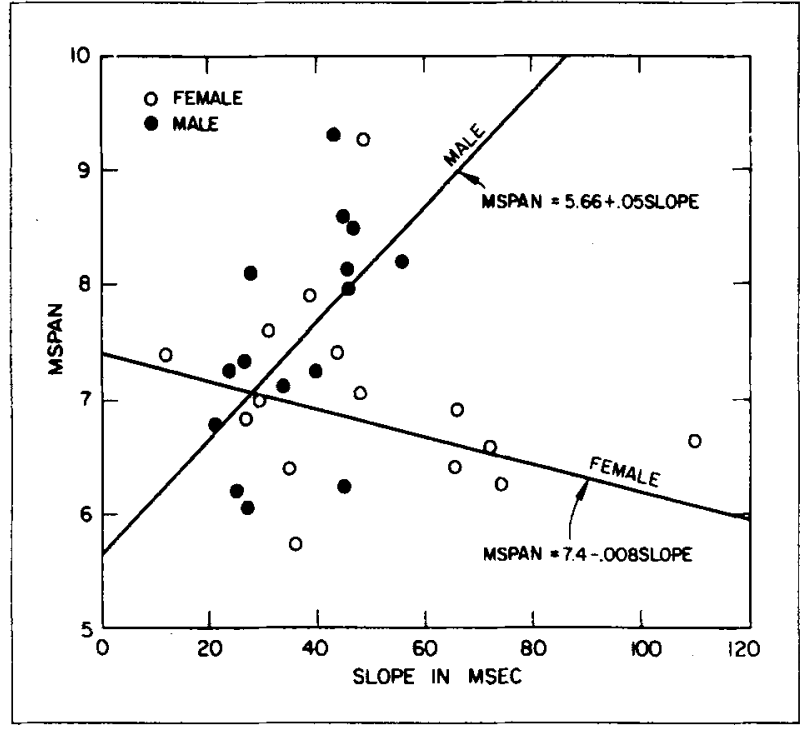

Figure 7. Scatter plot of SLOPE and MSPAN for male and female subjects. Lines fitted by least squares to the male and female observations.

obtained when data were analyzed separately for the male and female groups. Notice first from Table 7 that the squared multiple correlation $\left(\mathbf{R}^{2}\right)$ becomes much larger when predictions are made separately for the two sex groups. Second, compare the order in which predictors are entered into the three regression equations for SATM scores. For both sex groups, SLOPE is the best predictor, whereas for the group as a whole, the SLOPE parameter contributes almost nothing to the prediction. Thus, the large predictability of a variable can be totally obscured if the prediction is made without an awareness of the sex differences in the relationships between the dependent measure and the predictor. Next, examine the magnitude and the signs of the beta weights for these equations. For every variable, the signs of these weights are reversed for the male and female groups, but are the same within each sex group. In addition, the magnitudes of these weights are almost all greater for the groups by sex than for the total group.

\section{Discussion of Sex Differences}

The results of the analyses on sex differences shown in Tables 5, 6, and 7 present strong evidence that, for the sample in the present study, sex differences exist in the manner in which the cognitive processes under investigation are related to each other. To summarize these differences: (1) For female subjects, the higher the SATV and SATM scores, the slower the search rates; whereas for male subjects, the higher the SATV and SATM scores, the faster the search rates. (2) Male sub. jects with larger short-term memory capacities have slower search rates, whereas female subjects with larger short-term memory capacities have faster search rates. The multiple regression equations (see Table 7) also showed that different parameters contributed differently in magnitude and in direction to the prediction of SATV and SATM scores for the male and female samples. However, there is little sex difference in the means of any of these measures (see Table 2).

Because the present study was not designed to examine sex differences, we did not anticipate the differences in correlations and did not initially make provisions to use a larger sample of male and female subjects. The Ns used in the correlations were small $(\mathrm{N}=15)$; therefore, we would want to replicate this study with larger samples before making conclusive statements about and giving interpretations to differences in interrelationships of these cognitive measures between males and females. However, it is interesting to note that, for the present sample, large multiple correlations were obtained for the psychometric measures, using information processing parameters as predictors, when the data were analyzed separately for the male and female subjects.

Is there evidence from other studies for sex differences in the interrelationships of cognitive processes to support the results of the present study? A review of the research on sex differences in cognition (Garai \& Scheinfield, 1968; Maccoby \& Jacklin, 1974) indicates that studies of sex differences in cognitive functioning have primarily compared means and SDs of specific

Table 7

Stepwise Multiple Regression Prediction of SATV and SATM from Task Parameters

\begin{tabular}{|c|c|c|c|c|c|c|c|c|c|c|c|c|c|}
\hline & & \multicolumn{4}{|c|}{ All Subjects $\dagger$} & \multicolumn{4}{|c|}{ Female Subjects ${ }_{\dagger} \dagger$} & \multicolumn{4}{|c|}{ Male Subjects ${ }^{\dagger} \dagger$} \\
\hline & & 0 & $\mathbf{R}^{2}$ & $\Delta$ & $b$ & 0 & $\mathrm{R}^{2}$ & $\Delta$ & b & 0 & $\mathbf{R}^{2}$ & $\Delta$ & $\mathrm{b}$ \\
\hline \multirow[t]{2}{*}{ SATV } & $\begin{array}{l}\text { SLOPE } \\
\text { INT } \\
\text { MSPAN }\end{array}$ & $\begin{array}{l}1 \\
3 \\
2\end{array}$ & $\begin{array}{l}.08 \\
.11 \\
.11\end{array}$ & $\begin{array}{l}.08 \\
.00 \\
.03\end{array}$ & $\begin{array}{r}1.38 \\
.28 \\
-.77\end{array}$ & $\begin{array}{l}1 \\
2 \\
3\end{array}$ & $\begin{array}{l}.51 \\
.52 \\
.53\end{array}$ & $\begin{array}{l}.51 \\
.01 \\
.01\end{array}$ & $\begin{array}{r}3.42 \\
-.51 \\
-.39\end{array}$ & $\begin{array}{l}2 \\
1 \\
3\end{array}$ & $\begin{array}{l}.23 \\
.15 \\
.28\end{array}$ & $\begin{array}{l}.08 \\
.15 \\
.05\end{array}$ & $\begin{array}{r}-1.45 \\
1.57 \\
.93\end{array}$ \\
\hline & CONSTANT & \multicolumn{4}{|c|}{637} & \multicolumn{4}{|c|}{589} & \multicolumn{4}{|c|}{273} \\
\hline \multirow[t]{2}{*}{ SATM } & $\begin{array}{l}\text { SLOPE } \\
\text { INT } \\
\text { MSPAN }\end{array}$ & $\begin{array}{l}3^{*} \\
1 \\
2\end{array}$ & $\begin{array}{l}.04 \\
.04\end{array}$ & $\begin{array}{l}.04 \\
.00\end{array}$ & $\begin{array}{r}-1.00 \\
.15\end{array}$ & $\begin{array}{l}1 \\
2 \\
3^{*}\end{array}$ & $\begin{array}{l}.11 \\
.20\end{array}$ & $\begin{array}{l}.11 \\
.09\end{array}$ & $\begin{array}{r}1.60 \\
-1.25\end{array}$ & $\begin{array}{l}1 \\
3 \\
2\end{array}$ & $\begin{array}{l}.20 \\
.42 \\
.27\end{array}$ & $\begin{array}{l}.20 \\
.15 \\
.07\end{array}$ & $\begin{array}{r}-2.50 \\
1.79 \\
1.95\end{array}$ \\
\hline & CONSTANT & \multicolumn{4}{|c|}{722} & \multicolumn{4}{|c|}{680} & \multicolumn{4}{|c|}{357} \\
\hline
\end{tabular}

Note-Table includes squared multiple correlation $\left(R^{2}\right)$, increment to $R^{2}(\Delta)$, regression beta weights (b), and order (O) of variable entered into equation according to next largest partial correlation.

*Variable not included in regression equation because $F$ ratio $<.005$ 
cognitive measures. They have given insufficient consideration to the interrelationships among these descriptive measures. It was due to the scarcity of such correlational studies that Maccoby and Jacklin (1974) made a decision to review studies of mean differences only. Siegel (1965) found that there were no significant differences in performance on some cognitive tasks for girls and boys; however, emotional control was related positively to the boys' achievement and negatively to the girls' achievement. Even though Siegel related cognitive variables to affective variables, his study is similar to the present one in that the means showed no sex differences, while correlational analysis revealed differences between the male and female subjects. We conclude that future studies of sex differences in cognition need to attend to sex differences in the interrelationships among the cognitive measures.

Assuming the sex-difference results obtained in this study are real differences in cognitive functions between males and females, and not a characteristic peculiar to the present sample, what explanations can be given for these observed differences? One possible explanation is that sex differences in cognitive performance result from sex differences in personality or social factors. For example, high SATV and SATM females may be more cautious in performing the search tasks and, therefore, have slower search rates. Although we do not have any measures of cautiousness for these subjects, we do have a record of the number of errors that each subject made, which indicates how careful they were. The correlation between SATV and ERROR for females is -.40 and for males is -.14. A higher correlation is obtained for the females, but there is no difference in correlation for the two groups. Thus, there is no evidence that cautiousness relates differently to male and female cognitive performance.

Another possible explanation for the observed sex differences in the interrelationships of cognitive functions is that there are biochemical factors which cause sex differences in brain structure. For example, a sexlinked recessive gene for spatial ability has been documented by several studies (Bock \& Kolakowski, 1973; Hartlage, 1970; Stafford, 1961; Yen, 1975). Some researchers hypothesize that hormonal differences in the estrogen/androgen balance cause sex differences in verbal and spatial performance (Broverman, Klaiber, Kobayashi, \& Vogel, 1968; Petersen, Note 4). Harshman and Remington (Note 5) have reviewed clinical and experimental data from over 30 studies that indicate that "the brains of men and women tend to show differences in hemisphere specialization for language and spatial functions, with men showing a greater average degree of lateralization than women." Levy (1973) argues that the degree of lateralization would influence perceptual and verbal performance; that is, the less lateralized the brain, the greater interference between perceptual and verbal performance. Thus, sex differences in correlation among cognitive processes may result from sex differences in brain laterality. To examine such a hypothesis, laterality measures. can be obtained for subjects and correlated with other cognitive measures.

Our data do not present evidence to support any hypothesis relating to social or biological causes for sex differences in cognitive performance. These hypotheses do, however, suggest that there may be reasons to believe that the obtained sex-difference results in this study are real differences and not a random factor peculiar to our sample of male and female subjects. Further studies are necessary to verify these sex differences in the interrelationships of cognitive processes and to examine the causes for such differences. We recommend that future studies investigating individual differences in cognitive processes (1) use large and mixed samples of male and female subjects, (2) examine sex differences in the interrelationships of the cognitive variables, in addition to the comparison of means, and (3) include other noncognitive measures such as personality scales or laterality preference measures, which may relate to sex differences in cognitive differences.

In conclusion, the present study has demonstrated experimentally that, within the information processing theory framework of cognition, elemental component processes can be identified that are similar, or highly related, or the same for different cognitive tasks. Through well controlled laboratory investigations of individuals' performances on various cognitive tasks, we believe it is possible to further identify other similar or related processes and to arrive at a better understanding of the interrelationships of the components of the human cognitive system. Furthermore, our data suggest sex differences in the interrelationships of cognitive processes. We recommend that this difference be further examined.

\section{REFERENCE NOTES}

1. Rose, A. Human information processing: An assessment and research battery (Tech. Rep. 46). Ann Arbor, Mich: University of Michigan, Human Performance Center, 1974.

2. Lunneborg, C. E. Individual differences in memory and information processing (Tech. Rep.). Seattle, Wash: University of Washington, Educational Assessment Center. 1974.

3. Ward, W. C. Individual differences in information processing units (Bulletin RB-73-70). Princeton, N.J: Educational Testing Service, 1973.

4. Petersen, A. C. The relationship of androgenicity in males and females to spatial ability and fluent production. Unpublished doctoral dissertation, University of Chicago, 1973.

5. Harshman, R. A., \& Remington, R. Sex, language and the brain: $A$ review of the literature on adult sex differences in lateralization. Los Angeles, Calif: University of California, Phonetic Laboratory, 1975.

\section{REFERENCES}

ANDERson, J. R., \& Bower, G. H. Human associative memory. Washington, D.C: Winston, 1973. 
Atrinson, R. C., Holmgren, J. E., \& Juola, J. F. Processing time as influenced by the number of elements in a visual display. Perception \& Psychophysics, 1969, 6, 321-326.

Arkinson, R. C., \& Juola, J. F. Search and decision processes in recognition memory. In D. H. Krantz, R. C. Atkinson, and P. Suppes (Eds.), Contemporary developments in mathematical psychology. San Francisco: Freeman, 1974.

Atxinson, R. C., \& Shiffrun, R. M. Human memory: A proposed system and its control processes. In K. W. Spence \& J. T. Spence (Eds.), The psychology of learning and motivation (Vol. 2). New York: Academic Press, 1968.

Bieru, J., Bradburn, W., \& Galinsky, M. Sex differences in perceptual behavior. Journal of Personality, 1958, 26, 1-12.

Bock, R. D., \& Kolakowski, D. F. Further evidence of sexlinked major gene influence on human spatial visualizing ability. American Journal of Human Genetics, 1973, 25, 1-14.

Broverman, D. M., Klatber, E. L., Kobayashi, Y., \& VOGEL, W. Roles of activation and inhibition in sex differences in cognitive abilities. Psychological Review, 1968, 5, 23-50.

Carroll, J. Psychometric tests as cognitive tasks: A new "structure of intellect." In L. Resnick (Ed.), The nature of intelligence. Hilsdale, N.J: Lawrence Erlbaum/Halsted Press, 1976.

Cavanagh, J. P. Relation between the immediate memory span and the memory search rate. Psychological Review, 1972, 79, 525-530.

Chase, W. G., \& Simon, H. A. Perception in chess. Cognitive Psychology, 1973, 4, 55-81.

Cronbacr, L. J. The two disciplines of scientific psychology. The American Psychologist, 1957, 12, 671-684.

Estes, W. K., \& TAYLoR, H. A. A detection method and probabilistic models for assessing information processing from brief visual displays. Proceedings of the National Academy of Sciences, 1964, 52, 446-454.

Estes, W. K., \& TAYLoR, H. A. Visual detection in relation to display size and redundancy of critical elements. Perception \& Psychophysics, 1966, 1, 9-16.

Estes, W. K., \& Wessel, D. L. Reaction time in relation to display size and correctness of response in forced-choice visual signal detection. Perception \& Psychophysics, 1966, 1, 369-373.

Garai, J. E., \& Scheinfield, A. Sex differences in mental and behavioral traits. Genetic Psychology Monograph, $1968,77,169-229$.

GrLFORD, R., \& JuolA, J. Familiarity effects on memory search and visual search. Bulletin of the Psychonomic Society, 1976, 7, 142-144.
Hartlage, L. C. Sex-linked inheritance of spatial ability. Perceptual and Motor Skills, 1970, 3, 610.

Hunt, E. B., Frost, N., \& Lunneborg, C. E. Individual differences in cognition. In G. Bower (Ed.), The psychology of leaming and motivation (Vol. 7). New York: Academic Press, 1973.

Hunt, E. B., Lunneborg, C., \& Lewis, J. What does it mean to be high verbal? Cognitive Psychology, 1975, 7, 194-227.

LEVY, J. Lateral specialization of the human brain: Behavioral manifestations and possible evolutionary basis. In J. A. Kiger, Jr. (Ed.), The biology of behavior. Corvallis, Oreg: Oregon State University Press, 1973.

MACCOBY, E., \& JACKLIN, C. The psychology of sex differences. Stanford, Calif: Stanford University Press, 1974.

NeIsser, U. Cognitive psychology. New York: Appleton Century-Crofts, 1967.

Norman, D. A., \& Rumelhart, D. E. A system for perception and memory. In D. A. Norman (Ed.), Models of human memory. New York: Academic Press, 1970.

PosNer, H. I., \& Mitchell, R. F. Chronometric analysis of classification. Psychological Review, 1967, 74, 392-409.

SiEger, I. E. Rationale for separate analyses of male and female samples on cognitive tasks. Psychological Record, $1965,15,369-376$.

STERnBerG, $\mathbf{R}$. Intelligence, information processing and individual differences: $A$ componential analysis of analogical reasoning. Hillsdale, N.J: Lawrence Erlbaum, 1976.

STERNBERG, S. High-speed scanning in human memory. Science, 1966, 153, 652-654.

STERNBERG, S. Memory-scanning: Mental processes revealed by reaction-time experiments. American Scientist, 1969 , 57, $421-457$.

Stafford, R. E. Sex differences in spatial visualization as evidence of sex-linked inheritance. Perceptual and Motor Skills, 1961, 13, 428.

UNDERWOOD, B. J. Individual differences as a crucible in theory construction. American Psychologist, 1975, 30, 128-134.

Wescourt, K. T., \& Atkinson, R. C. Fact retrieval in human memory. In W. K. Estes (Ed.), Handbook of learning and cognitive processes (Vol. 4). Hillsdale, N.J: Lawrence Erlbaum, 1976.

YEN, W. M. Sex-linked major-gene influence on selected types of spatial performance. Behavior Genetics, 1975, 5, 281-298.

(Received for publication February 2, 1976; revision accepted May $10,1976$. ) 